\title{
Antibacterial Activity of Lactobacillus Strains Isolated from Mongolian Yogurt against Gardnerella vaginalis
}

\author{
Zhixiang Qian, Dan Zhao, Yu Yin, Hui Zhu $\mathbb{D}$, and Daijie Chen \\ College of Pharmacy, Shanghai Jiaotong University, Shanghai 201100, China \\ Correspondence should be addressed to Hui Zhu; julie19930@sjtu.edu.cn and Daijie Chen; cdj@sjtu.edu.cn
}

Received 27 November 2019; Revised 23 February 2020; Accepted 20 March 2020; Published 23 April 2020

Academic Editor: Fengjie Sun

Copyright ( 2020 Zhixiang Qian et al. This is an open access article distributed under the Creative Commons Attribution License, which permits unrestricted use, distribution, and reproduction in any medium, provided the original work is properly cited.

\begin{abstract}
Worldwide interest in the use of functional foods containing probiotic bacteria such as Lactobacillus and Bifidobacterium for health promotion and disease prevention has increased significantly. Probiotics have demonstrated beneficial properties including strengthening the body's natural defense system, inhibiting the growth of pathogenic bacteria, and regulating mental activity, but their effects on the human vagina have not been fully elucidated. The primary purpose of our study was to isolate Lactobacillus strains from old yogurt, a traditional dairy product, and investigate their probiotic potential with respect to the human vaginal system. Four Lactobacillus plantarum (L. plantarum) strains, named ZX1, ZX2, ZX27, and ZX69, were isolated from the yogurt samples. Simultaneously, we used a commercial Lactobacillus strain (Lactobacillus delbrueckii DM8909) as a control strain. We tested the antimicrobial activity of Lactobacillus isolates against Escherichia coli and Gardnerella vaginalis by agar spot and well diffusion tests. Then, we tested the antibiotic susceptibility of the 5 strains by using the minimal inhibitory concentration method. We attempted to detect possible bacteriocin genes by PCR sequencing technique. Using a chemically defined medium simulating genital tract secretions, we found that the selected Lactobacillus strains could alter the expression of known virulence genes in Gardnerella vaginalis. Bacteriocins derived from these isolated strains had potent antibacterial activity against G. vaginalis and E. coli, with the most effective activity observed in the case of ZX27. In addition, all strains including the L. delbrueckii DM8909 were positive for the presence of the plantaricin cluster of genes described in L. plantarum C11. The tested stains possessed the pln gene indicating that one of the antibacterial agents was plantaricin. We assume that the production of antimicrobial substances such as bacteriocins induce G. vaginalis to upregulate antimicrobial resistance genes. The new isolated strains have bacteriocin-related genes and can change the antimicrobial resistance gene transcription of G. vaginalis.
\end{abstract}

\section{Introduction}

Bacterial vaginosis (BV) is the most common cause of abnormal vaginal discharge in women of child-bearing age $[1,2]$. Although antimicrobial drugs, such as clindamycin and metronidazole, are recommended for the treatment of $\mathrm{BV}$, the use of these drugs is limited due to their side effects such as selecting of antibiotic-resistant pathogenic strains or causing superinfection [3]. Healthy vaginal flora is dominated by Lactobacillus that can lower the vaginal $\mathrm{pH}$ and/or produce metabolites such as hydrogen peroxide, lactic acid, and antibacterial molecules, including bacteriocins [4]. Lactobacillis plays a vital role in maintaining vaginal health. Lactobacillus species can act as biomarkers and agents that can promote various aspects of vaginal health [5]. G. vaginalis, a facultative anaerobe, is a leading harmful bacteria in BV [6]. Many studies have been carried out to investigate the dynamics between Lactobacillus and G. vaginalis in vitro and in vivo. The common characteristics of BV patients are the decrease of Lactobacillus and the increase of G. vaginalis in the vagina. $L$. rhamnosus GR-1 was the first probiotic used as a vaginal microbiota regulating Lactobacillus in 1988 [7]. It was demonstrated that $L$. crispatus could repress the expression of $v l y$ and sld genes in G. vaginalis $[8,9]$. Other researchers discovered that Lactobacillus could kill G. vaginalis and inhibit NF- $\kappa$ B activation, G. vaginalis-induced epithelial cell disruption, myeloperoxidase activity, and IL- $1 \beta$ and TNF- $\alpha$ expression in mice $[10,11]$. Clinical experiments also indicated that probiotics such as $L$. rhamnosus GR-1 and L. reuteri RC-14 have a therapeutic effect in reproductive tract infection 
[12]. L. plantarum is a common species in dairy products. Some researchers also reported that they have isolated $L$. plantarum from cheeses $[13,14]$ and that these isolates have an antibacterial activity against $L$. monocytogenes, E. coli, and S. enteritidis. Devi et al. [15] isolated L. plantarum subsp. plantarum MTCC 5422 from fermented cereal and discovered that MTCC 5422 effectively induced inflammatory conditions due to its anti-inflammatory activity. Except for G. vaginalis, E. coli remains the most common etiological organism for causing urinary tract infections (UTIs) [16].

Bacteriocins are small, heat-stable peptides which are produced by bacteria and are active against other bacteria [17]. There are two antibacterial mechanisms of bacteriocins: binding to lipid II and preventing cell wall synthesis, which can lead to cell death, or using lipid II as a docking molecule to initiate a process of membrane insertion and pore formation that leads to rapid cell death [18]. Bacteriocins have also been shown to be involved in immunoregulation. It was demonstrated that plantaricin EF produced by L. plantarum NCIMB8826 elevated TNF- $\alpha$ and IL-6 concentrations in mouse intestines [19].

Old yogurt is a traditional fermented food for herdsmen in Inner Mongolia, China, which is a solid yoghurt, and has longer fermentation process and more sour taste than the regular yogurt. Old yogurt only contains milk, sugar, or honey and a fermented culture. Yogurt is an important source of probiotics and has multiple beneficial effects on human health. For example, Lactobacillus bulgaricus was isolated from yogurt in 1905 [20]. Zhu et al. [21] showed that bioyogurt and the probiotics that it contains were capable of inhibiting specific periodontal pathogens. Yogurt with selected probiotic strains such as N1115 may reduce the risk of acute upper tract infections in the elderly [22].

The aim of our study was to isolate Lactobacillus strains from old yogurt and identify whether they had any potential activity against Gardnerella vaginalis.

\section{Materials and Methods}

2.1. Sample Collection, Strains, Cell Lines, and Growth Conditions. A total of 10 condensed yogurts were purchased from the shepherds in the Hulun Buir region of China. Then, the yogurts were shipped by cold-chain transportation to Shanghai Jiaotong University, Shanghai, for further experiments.

We grew Lactobacillus strains in de Man, Rogosa, and Sharpe (MRS) medium for $16-24 \mathrm{~h}$ at $37^{\circ} \mathrm{C}$ in an anaerobic system (RUSKINN, UK). Gardnerella vaginalis (ATCC49145) was purchased from the Guangdong culture collection center and cultured in brain-heart infusion (BHI) broth supplemented with yeast extract (1\%), maltose $(0.1 \%)$, glucose $(0.1 \%)$, and horse serum (10\%) (BHIS) at $37^{\circ} \mathrm{C}$ for $24 \mathrm{~h}$ under anaerobic conditions. Escherichia coli (ATCC 25922) was grown on Luria-Bertani medium (LB) for $12 \mathrm{~h}$ at $37^{\circ} \mathrm{C}$. Hela cells, a cervical epithelial cell line, were grown in Dulbecco's Modified Eagle's Media (DMEM) (HyClone, USA) supplemented with $10 \%$ fetal bovine serum (FBS) (Gibco, USA) and $1 \%$ antibiotics (penicillin and streptomycin) (Gibco, USA) at $37^{\circ} \mathrm{C}$ in a $5 \% \mathrm{CO}_{2}$ atmosphere.
2.2. Isolation and Identification of Lactobacillus Strains. MRS agar and broth were used to streak yogurt samples and enrich for the Lactobacillus strains. Colonies with typical Lactobacillus morphology, white in color and producing a fruity aroma, that were Gram positive were selected and inoculated in MRS broth. Ultimately, isolates were confirmed and identified by genetic analysis using PCR and $16 \mathrm{~S}$ rDNA sequencing. The genomic DNA of the Lactobacillus strains (from $2 \mathrm{~mL}$ of Lactobacillus culture) was extracted using a bacterial DNA isolation kit (Sangon Biotech, China). Universal PCR primers $27 \mathrm{~F}$ (AGAGTTTGATCCTGGCTCAG) and 1492R (TACGGC TACCTTGTTACGACTT) were used to amplify the $16 \mathrm{~S}$ rDNA gene. The PCR protocol was performed with the following thermal cycling parameters: $95^{\circ} \mathrm{C}$ for $10 \mathrm{~min}$ followed by 30 cycles of denaturation at $94^{\circ} \mathrm{C}$ for $30 \mathrm{~S}$, primer annealing at $60^{\circ} \mathrm{C}$ for $30 \mathrm{~s}$, elongation at $72^{\circ} \mathrm{C}$ for $1.5 \mathrm{~min}$, and thermal retardation at $72^{\circ} \mathrm{C}$ for $10 \mathrm{~min}$. Further, the PCR products were sequenced at the Invitrogen Biotechnology Company (Shanghai, China) and subjected to Basic Local Alignment Search Tool (BLAST) in the National Center for Biotechnology Information (NCBI). Finally, the phylogenic tree was constructed using the neighbor-joining method with MEGA 7 software using a bootstrap value of 1000. A commercial strain, Lactobacillus delbrueckii DM8909, isolated from Dingjunsheng (live Lactobacillus capsule for vaginal use $^{\circledR}$, Wanze Shuangqi) was used as the control.

\subsection{Antibacterial Tests In Vitro by Agar Spot and Well} Diffusion Tests. The antagonistic activity of the Lactobacillus strains against $G$. vaginalis and $E$. coli was investigated as previously described [23], with slight modification. In brief, for the spot agar test, a $2 \mu \mathrm{L}$ aliquot from each Lactobacillus strain suspension (at approximately $1 \times 10^{9} \mathrm{CFU} / \mathrm{mL}$ ) cultivated overnight in MRS broth was spotted on the surface of MRS agar containing $1.5 \%(w / v)$ agar and incubated for $24 \mathrm{~h}$ at $37^{\circ} \mathrm{C}$. A $100 \mu \mathrm{L}$ aliquot of $G$. vaginalis or E. coli suspension was then mixed with $100 \mathrm{~mL}$ of soft BHIS agar or LB agar $(0.7 \%$ agar) (final viable count of approximately 1 $\times 10^{6} \mathrm{CFU} / \mathrm{mL}$ ) and poured over the spot-inoculated MRS agar. The plates were incubated aerobically at $37^{\circ} \mathrm{C}$ for $48 \mathrm{~h}$. The antagonistic activity was determined by the diameter $(\mathrm{mm})$ of growth inhibition zones around each spot, corrected for the diameter of the spot. Uninoculated MRS agar was used as a negative control.

Generally, the antibacterial constituents produced by Lactibacillus were hydrogen peroxide, organic acids, and bacteriocins [24]. In order to examine the antibacterial mechanism, a well diffusion test was used as previously described [13]. The cultures were centrifuged $(8000 \times g, 20 \mathrm{~min})$ and sterile filtered $(0.22 \mu \mathrm{m})$. The supernatants were treated with catalase $\left(300 \mathrm{IU} / \mathrm{mL}, 37^{\circ} \mathrm{C}, 1 \mathrm{~h}\right.$, Macklin, China) and $1 \mathrm{M}$ $\mathrm{NaOH}$ (final pH6.5), in order to eliminate the effects of organic acid and hydrogen peroxide. These cell-free neutralized supernatants (CFN) were used as a bacteriocin solution and stored for further use. One hundred microliter aliquots of CFN were added into the $6 \mathrm{~mm}$ diameter holes on each agar plate. MRS broth was added into holes of the control group. After $2 \mathrm{~h}$, the liquid was volatilized, and the bacteriocins were permeated into the agar; then, $100 \mu \mathrm{L}$ of $G$. 
vaginalis $\left(1 \times 10^{6} \mathrm{CFU} / \mathrm{mL}\right)$ was spread evenly on the surface of the agar plates. Plates were incubated at $37^{\circ} \mathrm{C}$ for $48 \mathrm{~h}$, and the diameters of the inhibition growth zones were measured. All experiments were performed in triplicate.

\subsection{Antibacterial Testing of Untreated Cell-Free Supernatant} (CFS) and CFN. To determine the antibacterial activity of the $\mathrm{CFS}$ and CFN, G. vaginalis was grown at $37^{\circ} \mathrm{C}$ for $24 \mathrm{~h}$ in BHIS broth. The G. vaginalis culture was diluted with BHIS broth medium by $5 \%$ to $5 \times 10^{7} \mathrm{CFU} / \mathrm{mL}$. Then, $100 \mu \mathrm{L}$ of G. vaginalis suspension and $100 \mu \mathrm{L}$ of CFS and CFN were added to the wells of a 96-well microtiter plate in five replicates for each Lactobacillus CFS and CFN. One hundred microliters of BHIS broth and $100 \mu \mathrm{L}$ of CFS and CFN were added to the wells of a 96-well microtiter plate in three replicates for each Lactobacillus CFS and CFN as blanks. The plates were then incubated anaerobically at $37^{\circ} \mathrm{C}$ for $24 \mathrm{~h}$. In the control wells, the CFS or CFN was replaced by sterile MRS broth. The optical density (OD) at $595 \mathrm{~nm}$ was recorded after incubation using a microplate reader (Thermo Scientific 3001 , USA). The growth was calculated as the measured value minus the average blank value.

2.5. Antibiotic Susceptibility Testing. The minimum inhibitory concentrations (MIC) of cefoxitin sodium, ampicillin, kanamycin, gentamycin, erythromycin, tetracycline, polymyxin B, chloramphenicol, nalidixic acid, linezolid, metronidazole, and clindamycin against the selected Lactobacilli strains were determined using a broth microdilution test as previously described [25]. The plates were statically incubated at $37^{\circ} \mathrm{C}$ for $24 \mathrm{~h}$. Subsequently, the bacterial growth was visually observed, and the minimum inhibitory concentration of each antibiotic was confirmed as the lowest concentration capable of inhibiting visible bacterial growth.

2.6. Detection of the Plantaricin-Related Genes. The presence of plantaricin-related genes was investigated in five Lactobacillus isolates by PCR with specific primers, as previously described [26]. In addition, the entA, gasA, and laf genes were also detected using primers as previously reported [27-29]. All primers were designed based on published sequences and chemically synthesized by Invitrogen Biotechnology Company (Shanghai, China) (Supplementary Table S1). The PCR products were sent to Invitrogen Biotechnology Company (Shanghai, China) for sequencing (Supplementary Figure S1-S16). The obtained sequences were compared with known sequences in the NCBI database using the MegAlign software 8(DNASTAR, Inc., Madison, USA). If the similarity index was more than $90 \%$, the strain was positive for bacteriocin. If the similarity index was less than $90 \%$ or there were no PCR products, the strain was considered bacteriocin negative.

2.7. Virulence Genes in G. vaginalis Affected by Lactobacillus. To investigate interactions between Lactobacillus and G. vaginalis in Hela cell monolayers, we used a medium simulating genital tract secretions [8]. We studied two conditions for this. First, Hela cells were covered with Lactobacillus before infecting them with $G$. vaginalis (G. vaginalis infection in Hela with Lac). Second, G. vaginalis was allowed to adhere to Hela cells and then Lactobacillus was added (G. vaginalis infection in Hela treated with Lac). For the first condition, a Lactobacillus suspension, adjusted to $1 \times 10^{9} \mathrm{CFU} / \mathrm{mL}$, was added to a monolayer of Hela cells for $3 \mathrm{~h}$. Afterwards, the Hela cells were washed with PBS twice. Then, a G. vaginalis suspension, adjusted to the same concentration, was added to Hela cell monolayers pretreated with Lactobacillus for $3 \mathrm{~h}$. Next, the monolayer was washed with PBS three times. The G. vaginalis that adhered to Hela cells after $3 \mathrm{~h}$ served as a control. For the second condition, a G. vaginalis suspension was added to a monolayer of Hela cells for $3 \mathrm{~h}$ and then washed twice with PBS. The Lactobacillus suspension was then added to the Hela cells infected with G. vaginalis for $3 \mathrm{~h}$ and then washed with PBS three times. The G. vaginalis that adhered to Hela cells after $3 \mathrm{~h}$ served as a control. Total RNA from these two conditions was extracted using TRIzol (Beyotime, China) according to the manufacturer's instructions. RNA concentration and purity were determined with a Microplate Reader (BioTek, USA). Finally, the ReverTra Ace qPCR RT Kit (TOYOBO, Japan) was used to reverse transcribe $2 \mu \mathrm{g}$ of total RNA into cDNA.

Using qPCR, we examined the effect of Lactobacillus on the expression levels of three target genes (HMPREF0424 1122, HMPREF0424_0156, and HMPREF0424_0354) involved in antimicrobial resistance in G. vaginalis [30]. The cDNA was amplified by real-time quantitative PCR using SYBR Green Realtime PCR Master Mix (TOYOBO, Japan) on a StepOnePlus system (Applied Biosystems, USA) with primers listed in Supplementary Table S1. The real-time PCR conditions were as follows: $95^{\circ} \mathrm{C}$ for $1 \mathrm{~min}$, followed by 40 cycles of denaturation at $95^{\circ} \mathrm{C}$ for $15 \mathrm{~s}$, annealing at $60^{\circ} \mathrm{C}$ for $15 \mathrm{~s}$, and extension and fluorescent data collection at $72^{\circ} \mathrm{C}$ for $45 \mathrm{~s}$. For melting curve analysis, the temperature was decreased from $95^{\circ} \mathrm{C}$ to $65^{\circ} \mathrm{C}$ at a rate of $0.1^{\circ} \mathrm{C} / \mathrm{s}$ with continuous acquisition of the fluorescence signal intensity. Data were analyzed using Applied Biosystems software, and differences in mRNA expression levels were calculated after normalizing to the $16 \mathrm{~S}$ rRNA level. Results are expressed as the fold change relative to that in the control group based on ${ }^{\Delta \Delta \mathrm{Ct}}$ value analysis [31].

2.8. Statistical Analysis. Experimental results were analyzed for statistical significance using GraphPad Prism (GraphPad, San Diego, USA). Independent Student $t$-test analysis was performed. The statistical significance level was defined as $P<0.05$. All the results were expressed as the mean \pm SD.

\section{Results}

3.1. Isolation and Identification of Lactobacillus Strains. One hundred and five bacillus-shaped and Gram stain positive colonies were selected from ten yogurt samples and were then authenticated by $16 \mathrm{~S}$ rDNA gene sequencing. The 37 strains identified as Lactobacillus were selected for antibacterial tests against $G$. vaginalis in vitro by agar spot testing. Finally, four strains named ZX1, ZX2, ZX27, and ZX69 were selected for further study. Phylogenetic analysis of ZX1, ZX2, ZX27, and ZX69 showed high homology with L. plantarum (Figure 1). 


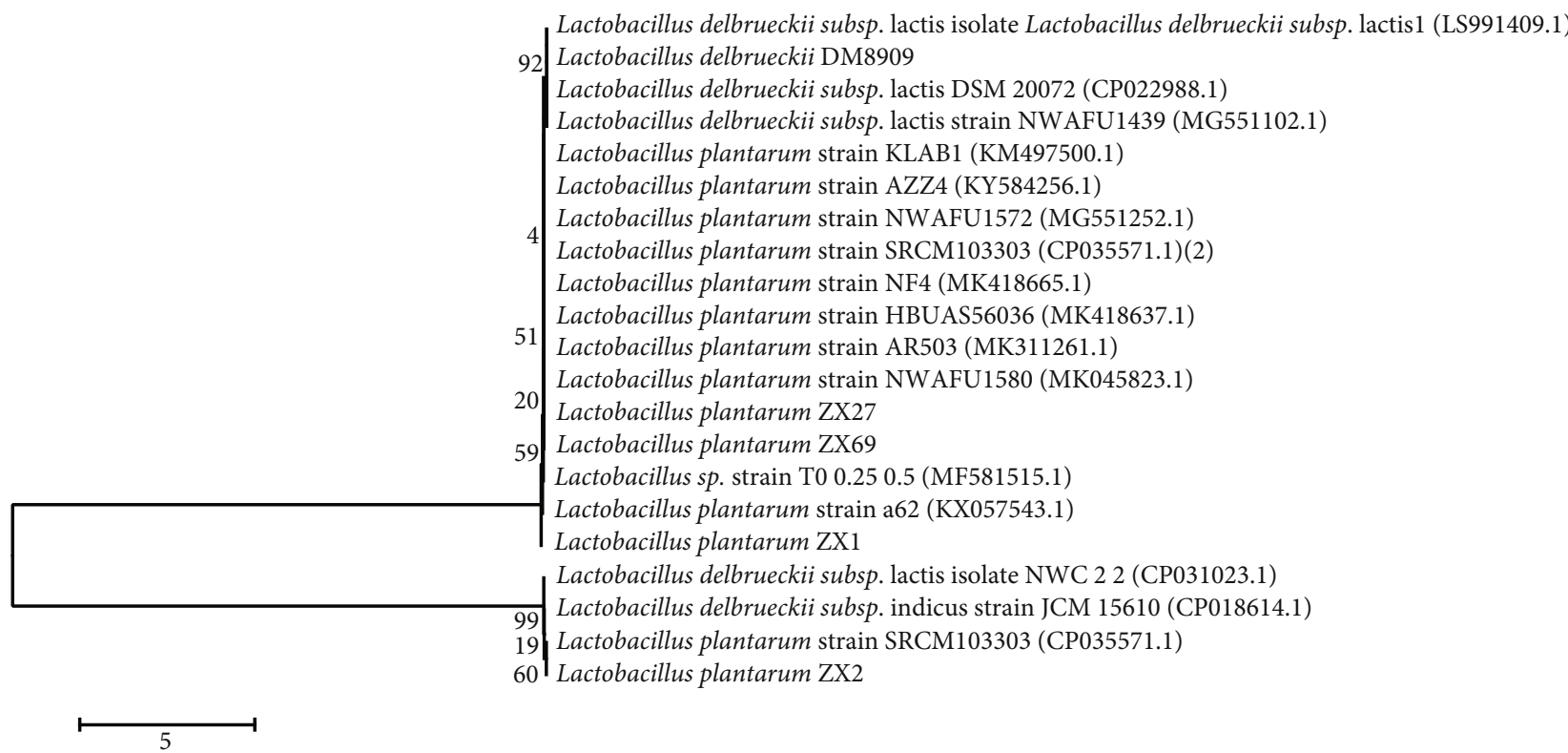

Figure 1: Phylogenetic analysis of strains of ZX1, ZX2, ZX27 ZX69, and DM8909 based on 16S rDNA partial gene sequences. The remaining sequences of the Lactobacillus in this figure were downloaded from NCBI.

3.2. Antibacterial Properties Determined In Vitro by Agar Spot and Well Diffusion Tests. The four new Lactobacillus strains isolated from old yogurts and a commercial strain DM8909 were screened for their antimicrobial activity by agar spot and well diffusion tests (Table 1). In the agar spot assay, the growth inhibition zone diameters with ZX1, ZX2, ZX27, and ZX69 were $\geq 10 \mathrm{~mm}$ for G. vaginalis and $\geq 20 \mathrm{~mm}$ for $E$. coli. The zones with DM8909 were $3.64 \mathrm{~mm}$ for G. vaginalis and $14.50 \mathrm{~mm}$ for E. coli, which were much lower values than those obtained with the four newly isolated Lactobacillus strains. Similarly, in a well diffusion test, the CFN of the four isolated Lactobacillus strains had inhibitory activity against $G$. vaginalis with growth inhibition zone diameters varying from 1.46 to $3.02 \mathrm{~mm}$. The CFN of DM8909 did not inhibit the growth of G. vaginalis. The most robust antagonistic activities for G. vaginalis in the agar spot and well diffusion assays were displayed by $L$. plantarum ZX27.

3.3. Antibacterial Testing of CFS and CFN against G. vaginalis. The CFS and CFN of the five Lactobacillis showed strong significant inhibitory effects (Figure 2 ) on the growth of $G$. vaginalis $(P<0.001)$. There was no significant difference in the potency of the inhibitory effect between the CFS from each of the five samples $(P>0.05)$. However, after neutralizing the supernatant acidity and hydrogen peroxide, the antimicrobial effect was significantly reduced $(P<0.01)$ compared with the respective CFS except for DM8909, which still showed a significant inhibition $(P<0.001)$ of $G$. vaginalis growth. The antimicrobial effect of CFN of DM8909 was significantly enhanced $(P<0.01)$ compared with the CFS, which was opposite to other four tested strains, indicating that acidity and hydrogen peroxide were not the main antimicrobial elements of the CFS from this strain against G. vaginalis.
3.4. Antibiotic Resistance. The studied Lactobacilli strains did not show resistance to erythromycin, linezolid, or clindamycin. Four out of the five strains were resistant to kanamycin and tetracycline (L. plantarum ZX1, ZX2, ZX27, and ZX69) and DM8909 was sensitive to kanamycin and tetracycline. Two out of the five strains were resistant to ampicillin (L. plantarum ZX27, ZX69) and chloramphenicol (L. plantarum ZX2, ZX27). All of the strains were resistant to cefoxitin sodium, gentamicin, polymyxin $\mathrm{B}$, nalidixic acid, and metronidazole (Table 2). Overall, the resistance profiles to antibiotics varied among the Lactobacillus strains. Resistance was determined according to the cut-offs recommended by the European Food Safety Authority (EFSA, 2012) [25].

3.5. Sequencing of the Bacteriocin Gene. In an attempt to determine whether the selected strains carried genes for the production of known plantaricins and other common bacteriocins, PCR analysis using primers specific for individual bacteriocin genes was used. All five strains were tested positive for $p \ln A, p \ln B, p \ln C, p \ln D, p \ln E F, p \ln I, p \ln J, p \ln K, p \ln G$, and $p \ln N$, suggesting that they could produce all the plantaricin peptides described in strain C11 [32]. None of the strains were positive for the plantaricins $\mathrm{NC} 8, \mathrm{~S}$, and $\mathrm{W}$. In addition, none of the strains were positive for genes encoding ent $A$, gas $A$, and laf, which are genes that are frequently found in Enterococcus faecalis, Lactobacillus casei, and/or lactic acid bacteria (Table 3).

3.6. Virulence Genes in G. vaginalis Are Affected by Lactobacillus. We used qPCR to evaluate and compare the effects on G. vaginalis cells after exposure to two Lactobacillus strains for $3 \mathrm{~h}$. Expression levels of three genes previously shown to be involved in antimicrobial resistance in G. vaginalis were compared to those in control untreated cells prepared under the same conditions without Lactobacillus. 
TABLe 1: Antimicrobial activity of Lactobacillus isolates determined by agar spot and well diffusion tests.

\begin{tabular}{lcccc}
\hline Strain & G. vaginalis ATCC49145 & \multicolumn{2}{c}{ E. coli ATCC25922 } \\
Well & $\begin{array}{c}\text { Well } \\
\text { diffusion } \\
\text { of CFN }\end{array}$ & Spot ager & $\begin{array}{c}\text { diffusion } \\
\text { of CFN }\end{array}$ \\
\hline ZX1 & $10.67 \pm 0.27$ & $2.94 \pm 0.32$ & $22.37 \pm 1.65$ & $<1( \pm 0.00)$ \\
ZX2 & $10.00 \pm 1.41$ & $1.56 \pm 0.13$ & $25.83 \pm 1.66$ & $1.38 \pm 0.1$ \\
ZX27 & $13.67 \pm 1.70$ & $3.02 \pm 0.43$ & $27.77 \pm 2.49$ & $<1( \pm 0.00)$ \\
ZX69 & $10.67 \pm 0.94$ & $1.46 \pm 0.22$ & $20.77 \pm 0.56$ & $2.52 \pm 0.65$ \\
DM8909 & $3.67 \pm 0.47$ & $<1( \pm 0.00)$ & $14.50 \pm 1.50$ & $<1( \pm 0.00)$ \\
\hline
\end{tabular}

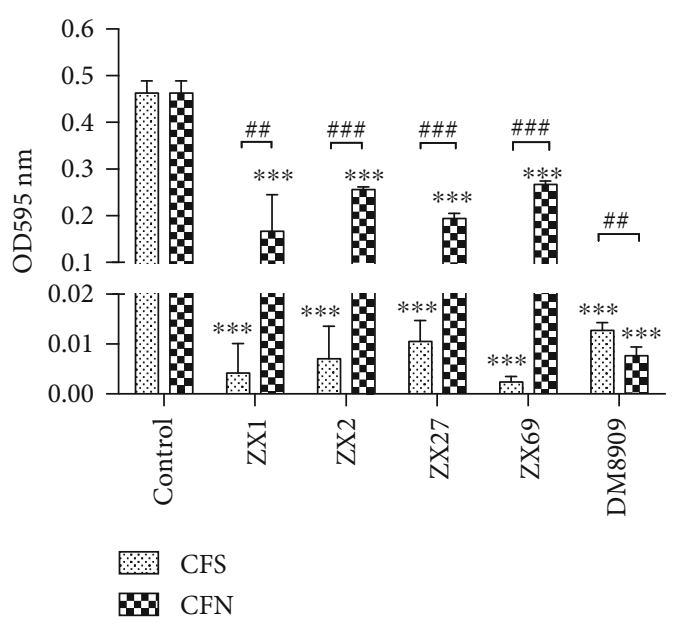

FIGURE 2: Gardnerella vaginalis growth in the presence of untreated cell-free supernatant (CFS) and cell-free neutralized supernatant (CFN). Control: $G$. vaginalis growth in BHIS broth added in $100 \mu \mathrm{L}$ sterile MRS broth. ${ }^{* * *} P<0.001$ compared with $G$. vaginalis growth in BHIS broth as the control.

TABLE 2: MIC of different antibiotics against the Lactobacillus stains.

\begin{tabular}{lccccc}
\hline \multirow{2}{*}{ Antibiotics } & \multicolumn{5}{c}{ Strains } \\
& ZX1 & ZX2 & ZX27 & ZX69 & DM8909 \\
\hline Cefoxitin sodium & $>512$ & $>512$ & $>512$ & $>512$ & $>512$ \\
Ampicillin & 32 & 32 & 64 & 128 & 1 \\
Kanamycin & $>512$ & $>512$ & $>512$ & $>512$ & 32 \\
Gentamycin & $>512$ & 512 & $>512$ & $>512$ & 512 \\
Erythromycin & 0.25 & 1 & 0.5 & $<0.25$ & $<0.25$ \\
Tetracycline & 256 & 256 & 512 & $128-256$ & $4-8$ \\
Polymyxin B & $>512$ & 512 & $>512$ & 512 & 128 \\
Chloramphenicol & 16 & 64 & 64 & $16-32$ & 8 \\
Nalidixic acid & $>512$ & $>512$ & $>512$ & $>512$ & $>512$ \\
Linezolid & 4 & 8 & 16 & $32-64$ & 4 \\
Metronidazole & $>512$ & $>512$ & $>512$ & $>512$ & $>512$ \\
Clindamycin & 0.5 & 0.5 & $<0.25$ & $<0.25$ & 0.5 \\
\hline
\end{tabular}

Among the three genes, HMPREF0424_0354 was not detected. Regardless of the conditions of Lactobacillus treatment, significant increases in the expression of HMPREF0424_0156 and HMPREF0424_1122 were observed in adherent G. vaginalis cells in the presence of Lactobacillus. Further, the effects of different Lactobacillus strains were variable for different genes. When G. vaginalis infected Hela cells pretreated with Lactobacillus, HMPREF0424_0156, which encodes bacitracin transport ATP-binding protein $B c r A$, was significantly upregulated by DM8909 (fourfold) and by ZX27 (20-fold) (Figure 3(a)). HMPREF0424_1122, which encodes a multidrug resistance $\mathrm{ABC}$ transporter, was also significantly upregulated by DM8909 (14-fold) and by ZX27 (13-fold). When G. vaginalis had already adhered to Hela cells, Lactobacillus also upregulated HMPREF0424_0156 by 30-fold (DM8909) and 5-fold (ZX27) (Figure 3(b)). Meanwhile, HMPREF0424_1122 was increased by 10-fold (DM8909) and four-fold (ZX27).

\section{Discussion}

$\mathrm{BV}$ is one of the most common diseases in women of childbearing age and is typically associated with the presence of the pathogenic bacteria, Gardnerella vaginalis. The female genital tract is dominated by Lactobacillus spp. in approximately $70 \%$ of women [5]. Lactobacillus species play an essential role in maintaining the ecosystem of the vagina [33-35]. Lactobacillus has proven to be efficient in treating BV by producing organic acids, hydrogen peroxide, bacteriocins, and adhesion inhibitors [34].

The Lactobacillus sp. used in this study was L. plantarum ZX1, ZX2, ZX27, and ZX69 and L. delbrueckii DM8909 (control strain). Four strains (L. plantarum ZX1, ZX2, ZX27, and ZX69) were isolated from yogurt samples and were identified as L. plantarum based on $16 \mathrm{~S}$ sequencing. The five tested Lactobacillus strains displayed the ability to inhibit the pathogenic bacteria $G$. vaginalis and $E$. coli. The antibacterial effects of L. delbrueckii DM8909 were weaker than the four new isolated strains in both agar spot and well diffusion tests, determined by the size of the zone of inhibition. In terms of DM8909, the inhibition diameter of CFN was $<1 \mathrm{~mm}$ and the inhibition diameter in the agar spot test was $3.67 \mathrm{~mm}$, both of which were much lower than the diameters of inhibition created by the four isolated Lactobacillus strains. Our findings were consistent with Wang et al.'s research [36], who reported that L. plantarum isolated from Tibetan yaks could strongly inhibit the growth of E. coli and S. aureus. Andreeva et al. [37] isolated a Lactobacillus sp. strain VLb3 from healthy Bulgarian women, which had a diameter of $12 \pm 1 \mathrm{~mm}$ in inhibition against G. vaginalis ATCC14018 by its CFS and CFN using well diffusion tests. In a study [38], three strains of Lactobacillus isolated from cocoa fermentation had an activity against G. vaginalis ATCC 49154 using the agar diffusion technique and the supernatant halos of L.plantarum 6.2 and L. plantarum 7.1 were 12 and $11 \mathrm{~mm}$, respectively. Another research showed that the CFS of some LAB had an inhibitory effect on the growth of $G$. vaginalis BCRC 17040 (inhibition diameter was about 2-3 mm) [39]. In above three research, the inhibition zone against G. vaginalis was about $3 \mathrm{~mm}$ after subtracting the well diameter, and the result in our study was 1.46 to $3.02 \mathrm{~mm}$. The results were abnormal for E. coli. The CFN of ZX1, ZX27, and 
TABLE 3: PCR-based detection of plantaricin genes and other bacteriocinogenic genes in L. plantarum strains and L. delbrueckii DM8909.

\begin{tabular}{|c|c|c|c|c|c|}
\hline \multirow[t]{2}{*}{ Bacteriocinogenic genes } & \multicolumn{5}{|c|}{ Strains } \\
\hline & ZX1 & $\mathrm{ZX} 2$ & ZX27 & ZX69 & DM8909 \\
\hline$p \ln A$ & + & + & + & + & + \\
\hline $\operatorname{pln} B$ & + & + & + & + & + \\
\hline$p \ln C$ & + & + & + & + & + \\
\hline$p \ln D$ & + & + & + & + & + \\
\hline$p \ln E F$ & + & + & + & + & + \\
\hline$p \ln I$ & + & + & + & + & + \\
\hline$p \ln J$ & + & + & + & + & + \\
\hline$p \ln K$ & + & + & + & + & + \\
\hline $\operatorname{pln} G$ & + & + & + & + & + \\
\hline$p \ln N$ & + & + & + & + & + \\
\hline Plantaricin NC8 structural gene & - & - & - & - & - \\
\hline Plantaricin S structural gene & - & - & - & - & - \\
\hline Plantaricin W structural gene & - & - & - & - & - \\
\hline entA & - & - & - & - & - \\
\hline $\operatorname{gas} A$ & - & - & - & - & - \\
\hline laf & - & - & - & - & - \\
\hline
\end{tabular}

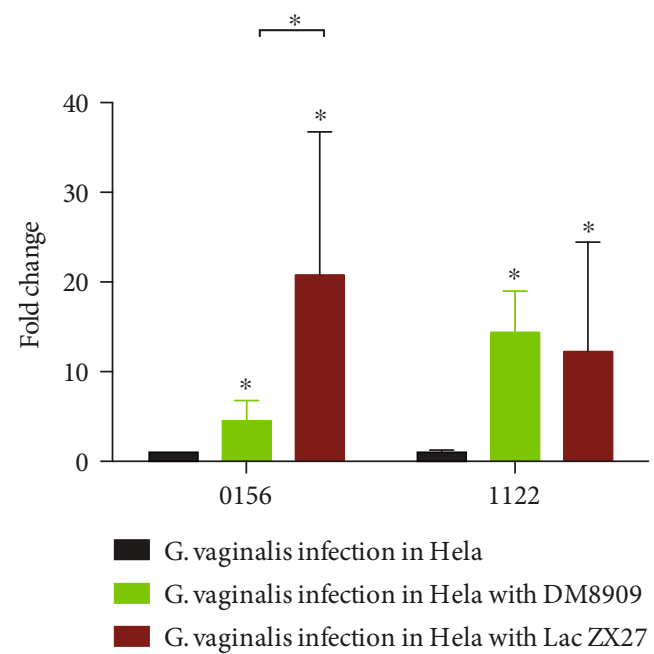

(a)

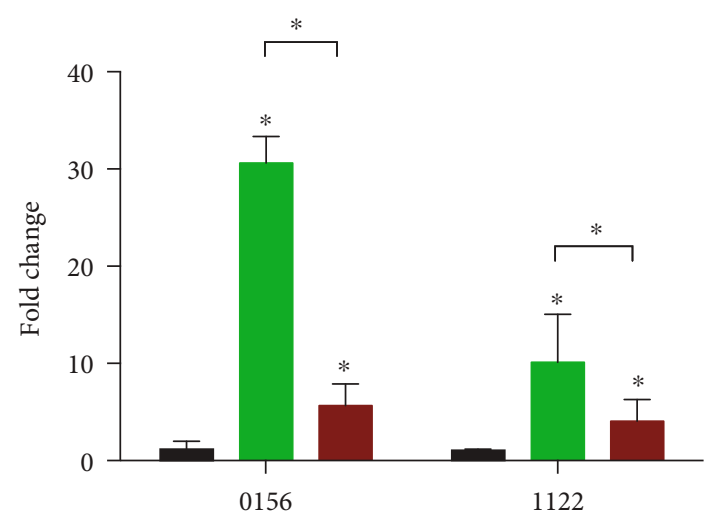

G. vaginalis infection in Hela

G. vaginalis infection in Hela treated with DM8909

G. vaginalis infection in Hela treated with Lac ZX27

FIgURE 3: Alterations in gene expression profiles associated with exposure of Lactobacillus, in (a) G. vaginalis infection in Hela with Lac and (b) G. vaginalis infection in Hela treated with Lac in each panel; fold change refers to the mean levels of gene expression across replicates calculated using the ${ }^{\Delta \Delta \mathrm{Ct}}$ method relative to levels in the untreated control. Fold change $=2^{-\Delta \Delta \mathrm{Ct}}$. Asterisks indicate statistically significant differences in the expression of each gene between treated samples and controls, as analyzed by one-way ANOVA with Dunnett's posttesting for multiple testing $\left({ }^{*} P \leq 0.05\right)$. Error bars indicate standard deviation.

DM8909 had no bacteriostatic activity. We thus speculated that the antibacterial properties of CFN from Lactobacillus have different antibacterial spectra.

The tested Lactobacillus strains caused a significant reduction in the microbial growth of G. vaginalis in BHIS broth, as determined by a change in the OD at $595 \mathrm{~nm}$. Jeong et al. also found that Lactobacillus kefiranofaciens DD2, isolated from kefir, can inhibit the growth of Sreptococcus mutans and Sreptococcus sobrinus using the same methods
[31]. At the same time, there was no significant difference between the CFS from the different Lactobacillus species regardless of their metabolic pattern. The facultative heterofermentative organism L. plantarum and the strictly homofermentative organism $L$. delbrueckii had similar antimicrobial effects (Figure 2). To determine the effect of bacteriocins produced by the tested Lactobacillus strains, the CFS was treated with $\mathrm{NaOH}$ and catalase to neutralize the $\mathrm{pH}$ and hydrogen peroxide $[13,23]$. It was observed that the neutralized CFS 
(CFN) caused a lower reduction in microbial growth than CFS, but CFN still showed a significant reduction in G. vaginalis growth when compared to the control. Wasfi et al. found that when CFS was treated to $\mathrm{pH}=7$, the ability of all 4 Lactobacillus inhibiting the growth of Streptococcus mutans is significantly declining $(P<0.01)$ [40]. Our results of the four L. plantarum are consistent with the results. DM8909 CFN had more significant growth inhibition than the CFS, suggesting that the main antimicrobial elements produced by DM8909 against $G$. vaginalis were bacteriocins. We assumed that the bacteriocins of DM8909 contribute to its antimicrobial effect at $\mathrm{pH}$ 6.5. Organic acids or hydrogen peroxide produced by the four new L. plantarum isolates ZX1, ZX2, ZX27, and ZX69 have antimicrobial effects on $G$. vaginalis. In the well diffusion assay, the inhibition diameter of DM8909 was less than $1 \mathrm{~mm}$, indicating that the CFN of DM8909 had no inhibition effects. Nevertheless, $100 \mu \mathrm{L}$ of this CFN inhibited the growth of G. vaginalis directly. One possibility for this discrepancy is that the inhibitory component of the DM8909 CFN could not diffuse in the agar plate.

For the four strains to be considered potential probiotics, they must be safe for human consumption. L. plantarum ZX27 and ZX69 were resistant to ampicillin, and L. plantarum ZX2 and ZX27 were resistant to chloramphenicol. All of the strains were resistant to cefoxitin sodium, gentamicin, polymyxin B, nalidixic acid, and metronidazole. The nature of this resistance warrants further studies before any of the strains can be considered safe for human use. In another study, three L. fermentum, which have anti-G. vaginalis activity, were sensitive to ampicillin and chloramphenicol [38]. Generally, Lactobacillus are safe probiotics, but some safety tests should be performed before using Lactobacillus in a clinical setting. In our study, the five Lactobacillus strains were resistant to the tested antibiotics.

All the CFN of the five strains have antimicrobial activities against $G$. vaginalis, indicating that the five strains can produce bacteriocins. Interestingly, the five stains in our study were positive for the genes $p \ln A, p \ln B, p \ln C, p \ln D$, $p \ln E F, p \ln I, p \ln J, p \ln K, p \ln G$, and $p \ln N$, but the inhibitory effects of different strains were different between the five strains. The phenomenon suggested that the antibacterial effects were strain specific. This may be because the gene expression patterns or bacteriocin secretion between these strains are different in the same culture conditions. Omar et al. isolated L. plantarum from ben saalga; however, they found only one strain (5.2.2) that possessed all the plantaricin genes, besides the $p \ln B$ gene [26].This is the first report that L. delbrueckii DM8909 has plantaricin genes. L. delbrueckii is the subspecies of Lactobacillus bulgaricus and is used in dairy fermentation broadly. It was unusual that L. bulgaricus possessed the $p \ln$ locus responsible for bacteriocin biosynthesis in L. plantarum C11, because the pln locus encoded plantaricins in L. plantarum [17, 32]. The reported Lactobacillus which have been found to harbor pln genes were all L. plantarum (C11, NC8, WCFS1, J23, and J51) [41].The result was consistent with the phylogenetic analysis in which the L. delbrueckii DM8909 was close to L. plantarum in the evolutionary system. Meanwhile, the CFN of DM8909, which contains bacteriocin, can inhibit G. vaginalis growth in BHIS broth directly.

Using an in vitro model, we tested gene expression in G. vaginalis after exposure to Lactobacillus. Remarkably, precoating the Hela monolayer with Lactobacillus or treating the cells with Lactobacillus after G. vaginalis had already adhered and enhanced the expression of the HMPREF0424 0156 and HMPREF0424_1122 transcripts. However, the degree of upregulation with the two Lactobacillus strains was different. In the precoating model, DM8909 upregulated HMPREF0424_0156 transcripts fourfold, whereas ZX27 increased the expression by 20 -fold $(P<0.05)$. In the second (treatment) model, DM8909 upregulated HMPREF0424 0156 and HMPREF0424_1122 to a greater extent than ZX27 $(P<0.05)$. This result reminds us that Lactobacillus will affect the gene expression in G. vaginalis and that different strains will have diverse regulatory effects.

\section{Conclusion}

The results of this study showed that the four new L. plantarum isolates could inhibit the growth of G. vaginalis, a causative agent of BV. The four new L. plantarum isolates and the commercial strain L. delbrueckii DM8909 were positive for genes related to plantaricins, which have antimicrobial activity. Our findings support that these L. plantarum strains could be used as probiotics for treating the BV disease $[42,43]$. Lactobacillus can upregulate the transcription levels of antimicrobial resistance genes in G. vaginalis. We speculated that the possible mechanism underlying this phenomenon is that bacteriocins produced by Lactobacillus induce altered gene transcription in G. vaginalis. Further studies, including survival rate in vaginal conditions and adhesion assay to VK2/E6E7 or Hela cells, should be conducted, as well as in vivo studies, to verify the potential health benefits of the new L. plantarum strains.

\section{Data Availability}

If requested, the original data of this article is available.

\section{Additional Points}

Research Involving Human Participants and/or Animals. This article does not contain any studies on either animals or human participants.

\section{Conflicts of Interest}

The authors declare that they have no conflicts of interest.

\section{Authors' Contributions}

Zhixiang Qian and Daijie Chen contributed to the design of the study, performed the experiments, analyzed the results, and wrote the manuscript. Dan Zhao collected samples and identified Lactobacillus strains. Yu Yin and Hui Zhu helped modify the paper. 


\section{Acknowledgments}

This experiment is a self-designed subject in the laboratory.

\section{Supplementary Materials}

Primer sequences used for PCR or RT-qPCR assay in the article and the gene electrophoretogram and sequences of bacteriocin. (Supplementary Materials)

\section{References}

[1] C. Primary, C. Sexual, and H. Workstream, "Bacterial vaginosis," Medicine, vol. 33, no. 10, pp. 58-61, 2007.

[2] M. E. Falagas, G. I. Betsi, and S. Athanasiou, "Probiotics for the treatment of women with bacterial vaginosis," Clinical Microbiology and Infection, vol. 13, no. 7, pp. 657-664, 2007.

[3] R. Palmeira-de-Oliveira, A. Palmeira-de-Oliveira, and J. Martinez-de-Oliveira, "New strategies for local treatment of vaginal infections," Advanced Drug Delivery Reviews, vol. 92, pp. 105-122, 2015.

[4] A. L. Servin, "Antagonistic activities of Lactobacilli and bifidobacteria against microbial pathogens," FEMS Microbiology Reviews, vol. 28, no. 4, pp. 405-440, 2004.

[5] M. I. Petrova, E. Lievens, S. Malik, N. Imholz, and S. Lebeer, "Lactobacillus species as biomarkers and agents that can promote various aspects of vaginal health," Frontiers in Physiology, vol. 6, 2015.

[6] A. W. Walker, "Studying the Human Microbiota," Microbiota of the Human Body, vol. 902, pp. 5-32, 2016.

[7] G. Reid, "The development of probiotics for women's health," Canadian Journal of Microbiology, vol. 63, no. 4, pp. 269-277, 2017.

[8] J. Castro, A. P. Martins, M. E. Rodrigues, and N. Cerca, "Lactobacillus crispatus represses vaginolysin expression by BV associated Gardnerella vaginalis and reduces cell cytotoxicity," Anaerobe, vol. 50, pp. 60-63, 2018.

[9] J. Castro, P. Alves, C. Sousa et al., "Using an _in-vitro_biofilm model to assess the virulence potential of Bacterial Vaginosis or non-Bacterial Vaginosis _Gardnerella vaginalis_isolates," Scientific Reports, vol. 5, no. 1, 2015.

[10] S.-E. Jang, J.-J. Jeong, S.-Y. Choi, H. Kim, M. Han, and D.-H. Kim, "Lactobacillus rhamnosus HN001 and Lactobacillus acidophilus La-14 attenuate Gardnerella vaginalis-infected bacterial vaginosis in mice," Nutrients, vol. 9, no. 6, p. 531, 2017.

[11] H. M. Joo, Y. J. Hyun, K. S. Myoung et al., "Lactobacillus johnsonii HY7042 ameliorates Gardnerella vaginalis-induced vaginosis by killing Gardnerella vaginalis and inhibiting NF- $\kappa \mathrm{B}$ activation," International Immunopharmacology, vol. 11, no. 11, pp. 1758-1765, 2011.

[12] R. Shamshu, J. Vaman, and C. Nirmala, "Role of probiotics in lower reproductive tract infection in women of age group 18 to 45 years," International Journal of Reproduction, Contraception, Obstetrics and Gynecology, vol. 6, no. 2, p. 671, 2017.

[13] A. Ołdak, D. Zielińska, A. Rzepkowska, and D. KołozynKrajewska, "Comparison of antibacterial activity of Lactobacillus plantarum strains isolated from two different kinds of regional cheeses from Poland: oscypek and korycinski cheese," BioMed Research International, vol. 2017, Article ID 6820369, 10 pages, 2017.
[14] G. Berta, V. Chebeñová, B. Brežná, D. Pangallq, L. Valík, and T. Kuchta, "Identification of lactic acid bacteria in Slovakian bryndza cheese," Journal of Food \& Nutrition Research, vol. 48, no. 2, pp. 65-71, 2009.

[15] S. M. Devi, N. K. Kurrey, and P. M. Halami, "In vitro antiinflammatory activity among probiotic Lactobacillus species isolated from fermented foods," Journal of Functional Foods, vol. 47, pp. 19-27, 2018.

[16] V. J. Mandracchia, D. W. Hayes, R. M. Yoho, and M. F. Hayes, "Diagnosis, differential and treatment options," Nature Reviews Microbiology, vol. 13, pp. 269-284, 2000.

[17] V. Kaškonienė, M. Stankevičius, K. Bimbiraitė-Survilienè et al., "Current state of purification, isolation and analysis of bacteriocins produced by lactic acid bacteria," Applied Microbiology and Biotechnology, vol. 101, no. 4, pp. 1323-1335, 2017.

[18] P. D. Cotter, C. Hill, and R. P. Ross, "Bacteriocins: developing innate immunity for food," Nature Reviews Microbiology, vol. 3, no. 10, pp. 777-788, 2005.

[19] X. Yin, D. Heeney, Y. Srisengfa, B. Golomb, S. Griffey, and M. Marco, "Bacteriocin biosynthesis contributes to the antiinflammatory capacities of probiotic Lactobacillus plantarum," Beneficial Microbes, vol. 9, no. 2, pp. 333-344, 2018.

[20] L. V. McFarland, "From yaks to yogurt: the history, development, and current use of probiotics," Clinical Infectious Diseases, vol. 60, Supplement 2, pp. S85-S90, 2015.

[21] Y. Zhu, L. Xiao, D. Shen, and Y. Hao, "Competition between yogurt probiotics and periodontal pathogens in vitro," Acta Odontologica Scandinavica, vol. 68, no. 5, pp. 261-268, 2010.

[22] F. Pu, Y. Guo, M. Li et al., "Yogurt supplemented with probiotics can protect the healthy elderly from respiratory infections: a randomized controlled open-label trial," Clinical Interventions in Aging, vol. 12, pp. 1223-1231, 2017.

[23] E. F. Garcia, W. A. Luciano, D. E. Xavier et al., "Identification of lactic acid bacteria in fruit pulp processing byproducts and potential probiotic properties of selected Lactobacillus strains," Frontiers in Microbiology, vol. 7, p. 1317, 2016.

[24] R. Vahedi Shahandashti, R. Kasra Kermanshahi, and P. Ghadam, "The inhibitory effect of bacteriocin produced by Lactobacillus acidophilusATCC 4356 and Lactobacillus plantarum ATCC 8014 on planktonic cells and biofilms of Serratia marcescens," Turkish Journal of Medical Sciences, vol. 46, no. 4, pp. 1188-1196, 2016.

[25] EFSA Panel on Additives and Products or Substances used in Animal Feed (FEEDAP), "Guidance on the assessment of bacterial susceptibility to antimicrobials of human and veterinary importance," EFSA Journal, vol. 10, no. 6, 2012.

[26] N. B. Omar, H. Abriouel, R. Lucas, M. Martínez-Cañamero, J.-P. Guyot, and A. Gálvez, "Isolation of bacteriocinogenic Lactobacillus plantarum strains from ben saalga, a traditional fermented gruel from Burkina Faso," International Journal of Food Microbiology, vol. 112, no. 1, pp. 44-50, 2006.

[27] M. Du Toit, C. M. A. P. Franz, L. M. T. Dicks, and W. H. Holzapfel, "Preliminary characterization of bacteriocins produced by Enterococcus faecium and Enterococcus faecalis isolated from pig faeces," Journal of Applied Microbiology, vol. 88, no. 3, pp. 482-494, 2000.

[28] G. Stoyancheva, M. Marzotto, F. Dellaglio, and S. Torriani, "Bacteriocin production and gene sequencing analysis from vaginal Lactobacillus strains," Archives of Microbiology, vol. 196, no. 9, pp. 645-653, 2014. 
[29] S. J. Macwana and P. M. Muriana, "A 'bacteriocin PCR array” for identification of bacteriocin-related structural genes in lactic acid bacteria," Journal of Microbiological Methods, vol. 88, no. 2, pp. 197-204, 2012.

[30] J. Castro, A. França, K. R. Bradwell, M. G. Serrano, K. K. Jefferson, and N. Cerca, "Comparative transcriptomic analysis of Gardnerella vaginalis biofilms vs. planktonic cultures using RNA-seq," NPJ Biofilms Microbiomes, vol. 3, no. 1, 2017.

[31] D. Jeong, D. H. Kim, K. Y. Song, and K. H. Seo, “Antimicrobial and anti-biofilm activities ofLactobacillus kefiranofaciensDD2 against oral pathogens," Journal of Oral Microbiology, vol. 10, no. $1,2018$.

[32] D. B. Diep, L. S. Håvarstein, and I. F. Nes, “Characterization of the locus responsible for the bacteriocin production in Lactobacillus plantarum C11," Journal of Bacteriology, vol. 178, no. 15, pp. 4472-4483, 1996.

[33] E. Amabebe and D. O. C. Anumba, "The vaginal microenvironment: the physiologic role of Lactobacilli," Frontiers in Medicine, vol. 5, pp. 1-11, 2018.

[34] G. Tachedjian, M. Aldunate, C. S. Bradshaw, and R. A. Cone, "The role of lactic acid production by probiotic Lactobacillus species in vaginal health," Research in Microbiology, vol. 168, no. 9-10, pp. 782-792, 2017.

[35] S. Borges, J. Silva, and P. Teixeira, "The role of Lactobacilli and probiotics in maintaining vaginal health," Archives of Gynecology and Obstetrics, vol. 289, no. 3, pp. 479-489, 2014.

[36] L. Wang, H. Zhang, M. U. Rehman et al., "Antibacterial activity of Lactobacillus plantarum isolated from Tibetan yaks," Microbial Pathogenesis, vol. 115, pp. 293-298, 2018.

[37] P. S. Patil, "International journal of advanced research in biological sciences," International Journal of Advanced Research in Biological Sciences, vol. 3, no. 7, pp. 181-192, 2016.

[38] W. F. B. Pessoa, A. C. C. Melgaço, M. E. de Almeida, L. P. Ramos, R. P. Rezende, and C. C. Romano, "In vitro activity of Lactobacilli with probiotic potential isolated from cocoa fermentation against Gardnerella vaginalis," BioMed Research International, vol. 2017, Article ID 3264194, 10 pages, 2017.

[39] C. C. Tsai, T. M. Lai, and Y. M. Hsieh, "Evaluation of Lactobacilli for antagonistic activity against the growth, adhesion and invasion of Klebsiella pneumoniae and Gardnerella vaginalis," Indian Journal of Microbiology, vol. 59, no. 1, pp. 81-89, 2019.

[40] R. Wasfi, O. A. Abd El-Rahman, M. M. Zafer, and H. M. Ashour, "Probiotic Lactobacillus sp. inhibit growth, biofilm formation and gene expression of caries-inducing Streptococcus mutans," Journal of Cellular and Molecular Medicine, vol. 22, no. 3, pp. 1972-1983, 2018.

[41] D. B. Diep, D. Straume, M. Kjos, C. Torres, and I. F. Nes, “An overview of the mosaic bacteriocin pln loci from Lactobacillus plantarum," Peptides, vol. 30, no. 8, pp. 1562-1574, 2009.

[42] P. Mastromarino, S. Macchia, L. Meggiorini et al., "Effectiveness of Lactobacillus-containing vaginal tablets in the treatment of symptomatic bacterial vaginosis," Clinical Microbiology and Infection, vol. 15, no. 1, pp. 67-74, 2009.

[43] P. B. Heczko, A. Tomusiak, P. Adamski et al., "Supplementation of standard antibiotic therapy with oral probiotics for bacterial vaginosis and aerobic vaginitis: a randomised, double-blind, placebo-controlled trial," BMC Women's Health, vol. 15, no. 1, pp. 1-12, 2015. 